\title{
Plastia valvar aórtica por ampliação de válvula com pericárdio bovino: nota prévia
}

\author{
Hércules Lisboa BONGIOVANI*, Paulo José de Freitas RIBEIRO*, Paulo Roberto Barbosa ÉVORA*, José \\ Carlos Franco BRASIL*, Celso Luis dos REIS*, Ricardo Nilsson SGARBIERI*
}

\section{RBCCV $44205-60$}

BONGIOVANI, H. L.; RIBEIRO, P. J. F.; ÉVORA, P. R. B.; BRASIL, J. C. F.; REIS, C. L.; SGARBIERI, R. N. - Plastia valvar aórtica por ampliação de válvula com pericárdio bovino: nota prévia. Rev. Bras. Cir. Cardiovasc., 3(2): 130-133, 1988.

RESUMO: Apresenta-se, preliminarmente, uma técnica para a correçāo do refluxo valvar aórtico pela ampliação de uma ou mais válvulas com pericárdio bovino. Após estudo experimental em peças animais isoladas, a técnica foi empregada, com sucesso, em uma paciente.

DESCRITORES: valvas cardíacas, cirurgia; próteses valvulares cardíacas, cirurgia; próteses valvulares cardiacas, biológicas.

\section{INTRODUÇĀO}

A correçāo cirúrgica da insuficiência aórtica é motivo de grande controvérsia. Esta particularidade fundamenta-se, principalmente, no fato de o miocárdio tolerar bem esta lesão valvar e o seu tratamento cirúrgico implica, quase sempre, na substituição da valva por prótese. As cirurgias reparadoras têm apresentado resultados pouco animadores, principalmente pelas dificuldades técnicas devidas às características anatômicas da valva aórtica, cujos mecanismos de fechamento são mais precisos do que aqueles envolvidos na dinâmica das valvas mitral e tricúspide. As valvas atrioventriculares apresentam maior superfície de coaptação e estruturas subvalvares, o que facilita a realização de correçōes plásticas, em caso de insuficiência².

Com base em uma razoável e bem sucedida experiência no tratamento cirúrgico da insuficiência mitral ampliando-se a cúspide posterior com pericárdio bovino ${ }^{3}$, tentou-se a adaptação desta técnica para a correção do refluxo valvar aórtico, ampliando-se uma ou mais de suas válvulas e associando-se, ou não, a elevaçāo de suas comissuras. Após estudo experimental em peças animais isoladas, a técnica foi empregada, com sucesso, em um paciente.

\section{CASUÍSTICA E MÉTODOS}

A técnica cirúrgica consta, basicamente da ampliação de uma ou mais válvulas da valva aórtica utilizan. do-se pericárdio bovino. A esta ampliação pode associar-se, ou não, elevação de suas bordas a nível das comissuras. Após a abertura da raíz da aorta e a infusāo direta da solução cardioplégica nos óstios coronarianos, procede-se à cuidadosa observação da valva como um todo, definindo possiveis aumentos do anel, retração ou desabamento de válvulas ou existência de lesōes associadas. Unindo-se as válvulas por tração com auxílio de pinças ou pontos de reparo, procede-se à desinserção da válvula mais retraída, até bem próximo das comissuras. Sutura-se, a seguir, um pequeno retalho de pericárdio bovino de forma oval e, aproximadamente, da mesma largura da válvula, no espaço deixado pela desinserçāo (Figura 1).

\footnotetext{
Trabalho realizado no Hospital do Coraçăo de Ribeirão Preto. Fundação Waldemar B. Pessoa. Ribeirão Preto, SP, Brasil. Recebido para publicaçăo em 21 de agosto, 1988.

- Do Hospital do Coração de Ribeirão Preto. Fundação Waldemar B. Pessoa

Endereço para separatas: Hércules Lisboa Bongiovani. Rua Rui Barbosa, 455, Apt: 140. 14015 Ribeirão Preto, SP. Brasil.
} 
BONGIOVANI, H. L.; RIBEIRO, P. J. F.; EVORA, P. R. B.; BRASIL, J. C. F.; REIS, C. L.; SGARBIERI, R. N. - Plastia valvar aórtica por ampliação de válvula com pericárdio bovino: nota prévia. Rev. Bras. Cir. Cardiovasc., 3(2): 130-133, 1988.

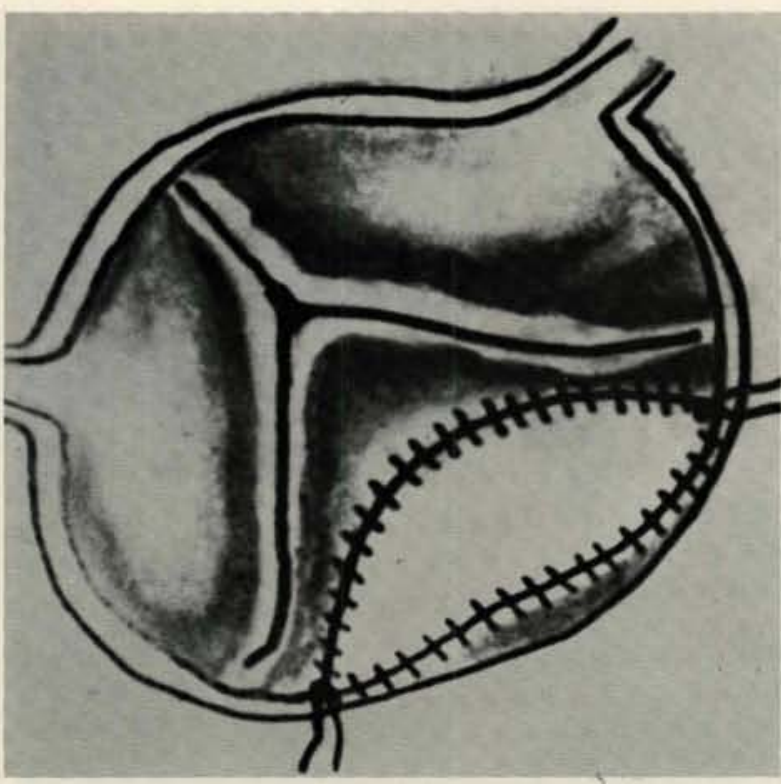

Fig. 1 - Representaçăo esquemática mostrando a ampliaçăo da válvula nāo coronariana.

A técnica descrita foi utilizada, com sucesso, em uma paciente de 24 anos, do sexo feminino, que foi encaminhada com quadro de insuficiência cardiaca grau III da NYHA. Ao exame físico, apresentava duplo sopro nos focos aórtico e mitral. O cateterismo cardíaco e o ecocardiograma revelaram a presença de dupla lesão mitral grau IV e insuficiência aórtica Gráu III, com gradiente transvalvar aórtico de $16 \mathrm{mmHg}$. Durante o ato cirúrgico, observou-se um discreto alargamento do anel aórtico, um discreto espessamento e retração das válvulas, permitindo um refluxo central. A lesão aórtica foi corrigida pela ampliação da válvula nāo coronariana, conforme a técnica descrita, elevando-se as bordas livres das válvulas com pontos de polipropileno ancorados em Teflon. A insuficiência mitral foi corrigida por cerclagem do anel posterior com uma fita de pericárdio bovino. Após a saída de circulação extracorpórea e total recuperação dos batimentos cardíacos, constatou-se a ausência de refluxo aórtico e discreta insuficiência mitral. No período de internação hospitalar, não apresentou sopros aórticos, a segunda bulha era normal à asculta, permanecendo um leve sopro sistólico no foco mitral. O ecocardiograma, realizado após 145 dias, mostrou insuficiência aórtica e mitral discretas (Figura 2). O estudo hemodinâmico mostrou insuficiência aórtica Grau I, ausência de insuficiência mitral e diminuição das câmaras cardiacas esquerdas, com gradiente transvalvar aórtico de $18 \mathrm{mmHg}$ (Figura 3).

\section{COMENTÁRIOS}

Desde o início da década de 50 , tem-se procurado um método eficaz para o tratamento cirúrgico da insufi-

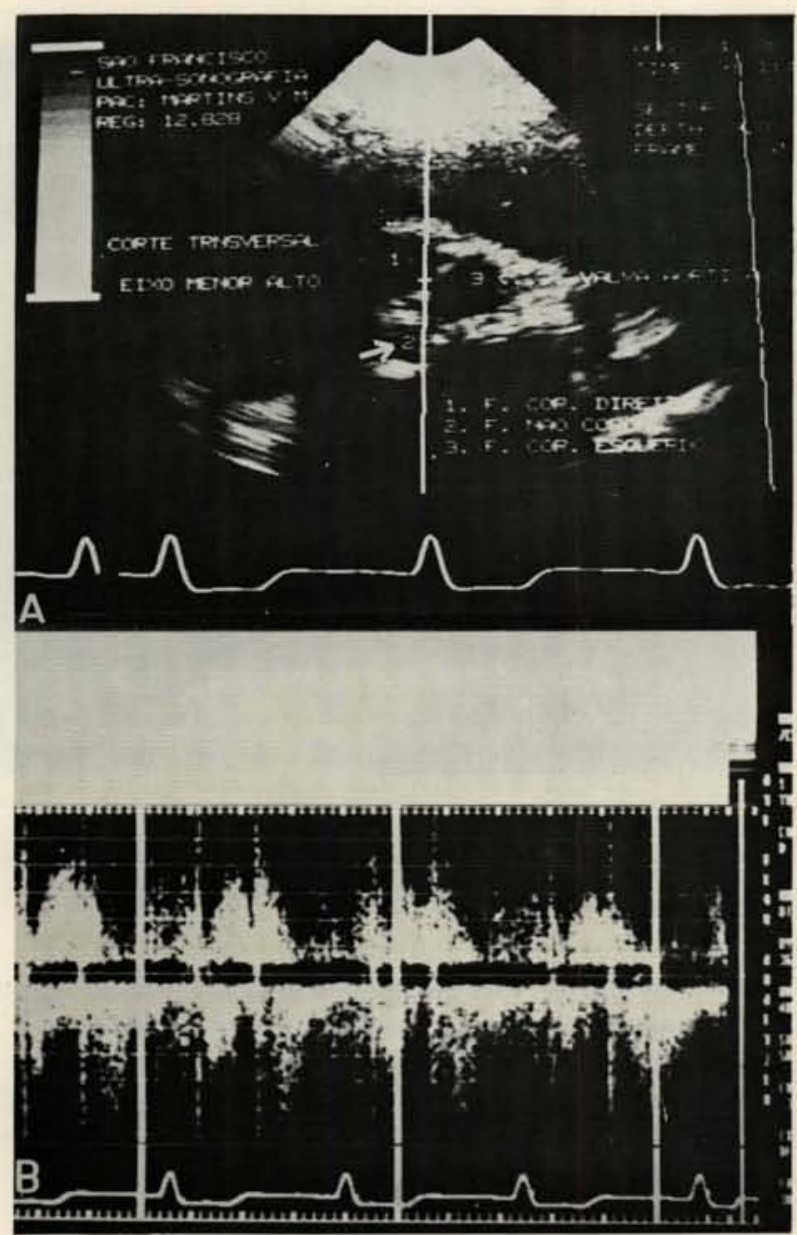

Fig. 2 - Ecocardiograma bidimensional e "Doppler" aórtico mostrando a boa coaptação das válvulas e ausência de refluxo valvar importante.

ciência aórtica, sendo que a técnica mais utilizada, atualmente, é a substituição valvar por prótese.

Os vários tipos de operaçōes conservadoras foram abandonados, por apresentarem maus resultados tardios. Algumas delas merecem citação: 1) diminuiçāo do diâmetro do anel valvar, procurando um melhor contato das válvulas na diástole ventricular ${ }^{1}{ }^{8}$; 2) remoção da válvula não coronariana e sutura das comissuras das outras duas e quando há dilatação do anel e deformidade minima ${ }^{4} ; 3$ ) sutura de prótese rígida de metilmetacrilato na aorta descendente, logo após a emergência da artéria subclávia ${ }^{5}$; 4) substituição de uma ou mais válvulas por outras artificiais de Teflon ou Dacron ${ }^{6.7}$; 5 ) diminuição da dilatação anular por sutura circular ${ }^{1}$; 6) desbridamento e descalcificação das válvulas retraídas. $O$ fator mais importante da correção cirúrgica conservadora da insuficiência aórtica valvar é a análise criteriosa da causa da lesão, procurando evidenciar um ou mais fatores: alargamento do anel valvar, desabamento das válvulas, válvulas de diferentes tamanhos, perda de tecido, principalmente na região das comissuras, e se a valva é bivalvulada $^{2}$. A ampliação de uma ou mais válvulas tornaria 
BONGIOVANI, H. L.; RIBEIRO, P. J. F.; ÉVORA, P. R. B.; BRASIL, J. C. F.; REIS, C. L.; SGARBIERI, R. N. - Plastia valvar aórtica por ampliação de válvula com pericárdio bovino: nota prévia. Rev. Bras. Cir. Cardiovasc., 3(2): 130-133, 1988.

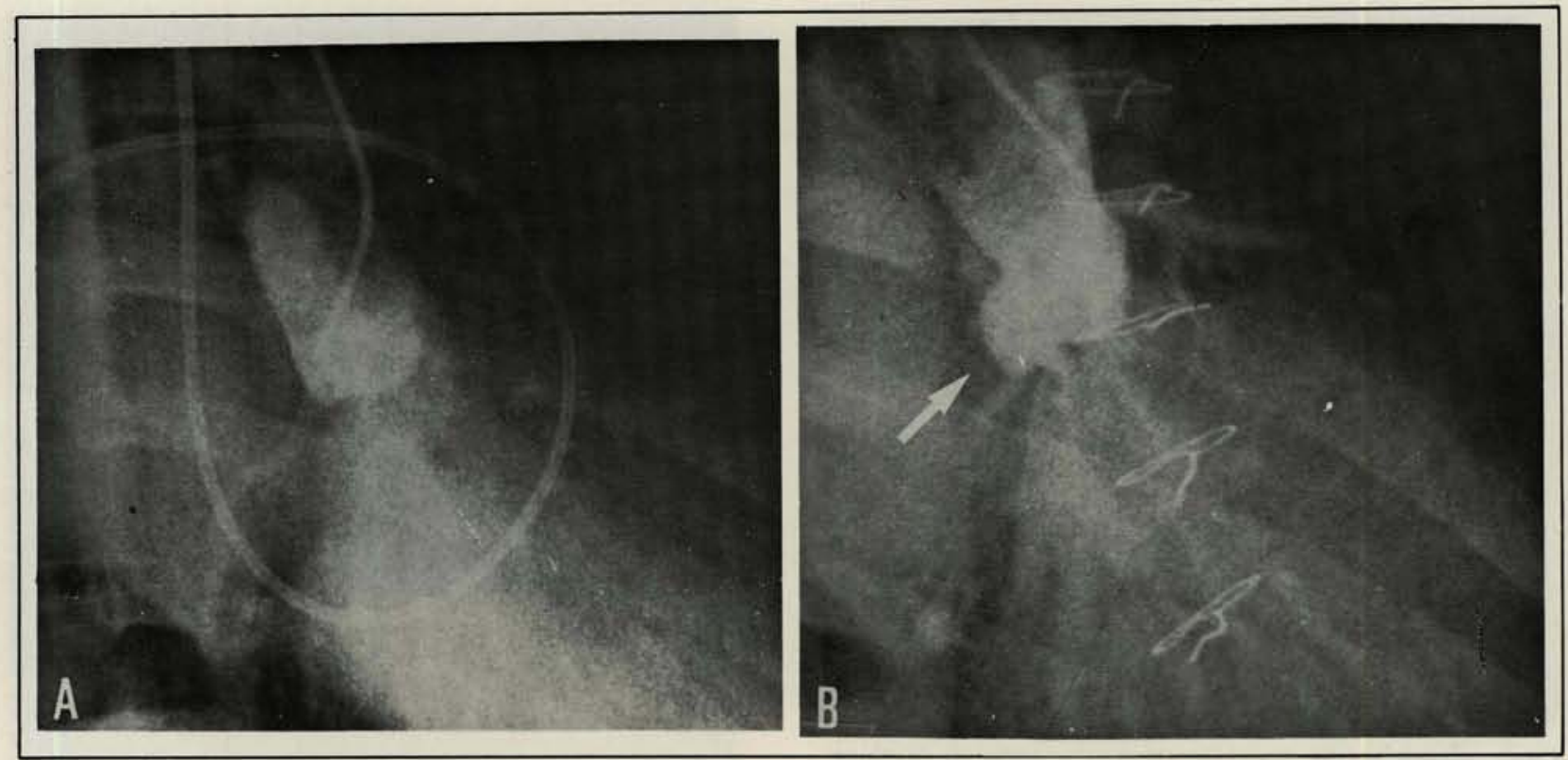

Fig. 3-Aortografia A = Pré-operatória: grande refluxo valvar; $\mathrm{B}=$ Pós-operatória: mostrando minimo refluxo valvar e a deformaçăo da válvula năo coronariana ampliada.

a valva suficiente, nas circunstâncias de retração e/ou desabamento. A associação da elevação da comissura é recurso auxiliar às vezes obrigatório, com o inconveniente de ficar certo gráu de gradiente transvalvar. A preocupação de deiscência na sutura do pericárdio é atenuada pelo espessamento do tecido na região de sua implantação, existente nos casos de doença reumática.

A técnica tem por objetivo tornar a valva suficiente, sem importantes disfunções ou gradientes transvalvares. No caso apresentado, a cirurgia implicou em uma deformação da válvula não coronariana ampliada (Figura 3), sem, contudo, criar obstáculos importantes ao esvazia- mento ventricular esquerdo, uma vez que o estudo hemodinâmico mostrou um gradientes de apenas $18 \mathrm{mmHg}$.

O objetivo principal desta divulgação é o de apresentar a proposição da técnica cirúrgica para a consideração de outros cirurgiōes e trazer subsídios para discussōes, no tocante à restauraçāo plástica da valva aórtica. A evolução do caso e a possibilidade de reprodução da técnica é, evidentemente, fundamental para uma apreciação mais definitiva.

Uma revisão bibliográfica, tão ampla quanto possível, não revelou nenhuma descrição semelhante à técnica cirúrgịca proposta de reconstruçāo das válvulas com pericárdio bovino. Portanto, esta poderá tratar-se de uma contribuição original.

BONGIOVANI, H. L.; RIBEIRO, P. J. F.; ÉVORA, P. R. B.; BRASIL, J. C. F.; REIS, C. L.; SGARBIERI, R. N. - Reparative operation for aortic valve incompetence by leaflet advancement with bovine pericardial: preliminary report. Rev. Bras. Cir. Cardiovasc., 3(2): 130-133, 1988.

ABSTRACT: A new technique for correction of aortic incompetence by the advancement of one more valve leaflets with bovine pericardial is presented. After experimental studies in isolated animal hearts this technique was used with success in one patient.

DESCRIPTORS: heart valves, surgery; heart valves, biologic. 
BONGIOVANI, H. L.; RIBEIRO, P. J. F.; ÉVORA, P. R. B.; BRASIL, J. C. F.; REIS, C. L.; SGARBIERI, R. N. - Plastia valvar aórtica por ampliação de válvula com pericárdio bovino: nota prévia. Rev. Bras. Cir. Cardiovasc., 3(2): 130-133, 1988.

\section{REFERÊNCIAS BIBLIOGRÁFICAS}

1 BAILEY, C. P. - Surgery of the heart. Philadelphia, Lea \& Febiger, 1955.

2 CARPENTIER, A. - Cardiac valve surgery: the french correction. J. Thorac. Cardiovasc. Surg., 86(3): $323-337,1983$.

3 ÉVORA, P. R. B.; RIBEIRO, P. J. F.; BRASIL, J. C. F.; OTAVIANO, A. G.; REIS, C. L.; BONGIOVANI, H. L.; BOMBONATO, R.; FERRAZ, M. A.; MENARDI, A. C.; SGARBIERI, R. N. - Experiência com dois tipos de técnicas para o tratamento cirúrgico da insuficiência mitral: I. Prótese com preservação de elementos do sistema valvar. II. Plastia valvar com reconstrução e avanço da cúspide posterior. Rev. Bras. Cir. Cardiovasc., 3(1): 36-49, 1988.

4 CARAMELla, J. J.; ANDERSEN, J. G.; OROPEZA, R. - The surgical treatment of aortic insufficiency by open plastic revision of the tricuspid aortic valve to a bicuspid valve. Surg. Gynecol. Obstet., 106(6): 679-686, 1958.
5 HUFNAGEL, C. A. - Direct approaches for the treatment of aortic insufficiency. Am. Surg., 25(5): 321-327, 1959.

6 HUfNAGel, C. A.; HARVEY, W. P.; RABIL, P. J.; Mc DERMOTT, T. F. - Surgical correction of aortic insufficiency. Surgery, 35(5): 673-683, 1954.

7 KAY, F. B.; MENDELSOHN, D.; SUZUKI, A.; ZIMMERMAN, H. - Correction of aortic regurgitation using plastic valves. JAMA, 176(13): 1077-1081, 1961.

8 TAYLOR, W. J.; THROWEN, W. B.; BLACK, H.; HARKEN, D. E. - The surgical correction of aortic insufficiency by circunclusion. J. Thorac. Surg., 34(2): 192-205. 1958.

AGRADECIMENTOS: Aos Drs. Antônio Luís Secches e Luís Antônio P. Finzi, pela documentação angiocardiográfica. Ao Dr. Jorge René G. Arévola, pela documentação ecocardiográfica. 